\title{
Disentangling distance decay of similarity from richness gradients: response to Soininen et al. 2007
}

\author{
Andrés Baselga \\ A. Baselga (baselga@mncn.csic.es), Dept de Biodiversidad y Biología Evolutiva, Museo Nacional de Ciencias Naturales - CSIC, \\ clJosé Gutiérrez Abascal, 2, ES-28006 Madrid, Spain.
}

Although great effort has been devoted to the description of richness patterns (see, as most recent examples, Svenning and Skov 2007, Montoya et al. 2007, Reyjol et al. 2007, Whittaker et al. 2007), much less literature on large scale beta diversity patterns is available (but see Qian et al. 2005). In a recent contribution, Soininen et al. (2007) performed an interesting meta-analysis searching for relationships between different predictors and the decay of similarity with geographic distance across a wide range of organisms, ecosystems and geographical gradients. Though the aim, objectives and conceptual background of this paper are very interesting, ecologists should be aware of strong drawback in their analytical framework that could potentially invalidate their major conclusions. One of their most remarkable results is the finding of a faster distance decay of similarity at higher latitudes, contradicting all current data and disagreeing Rapoport's rule, which predicts a positive correlation between species range size and latitude. I show here how the result may be produced by the confusing effect of richness gradients combined with the selection of a similarity measure inappropriate for the hypothesis tested. Soininen et al. (2007) sought to describe beta diversity patterns based on Sorensen similarity measure, which is known to be strongly biased by richness patterns (Koleff et al. 2003). Soininen et al. (2007: p. 8) acknowledged that this characteristic of the Sorensen metric could be a problem for the interpretation of their results when discussing the relation between similarity decay and latitude. In fact, there is a problem in all their tests involving variables covariating with richness (as trophic position or body weight, for example), because richness gradients distort turnover patterns if the similarity measure (Sorensen in this case) incorporates differences in richness as differences in composition. The solution would be to select a measure of beta diversity not influenced by richness differences.

\section{Conceptual background}

Why the Sorensen index is inappropriate in this case? It is well known the different performance of the great variety of available similarity indices (see, for example, Wilson and Shmida 1984, Koleff et al. 2003). The selection of one of these measures depends on the hypothesis to be tested. For the point raised here, two categories are relevant: those indices incorporating richness differences as compositional differences (i.e. Sorensen) and those yielding similarity values independent of richness variation (i.e. Simpson). Trivially, different richness implies different species composition, so it could be defended that richness differences should be taken into account by the selected similarity index. However, when richness gradients are present among the analyzed samples, the acceptance of richness differences as if they were composition differences results in the mixture of two different processes in the same measure, and thus the possible assumption of two different situations as equivalent. Suppose we analyze two pairs of samples (A, B, C, D) with 20, 20, 20 and 5 species, respectively. And suppose that $\mathrm{A}$ and $\mathrm{B}$ share 8 species (therefore 12 species are exclusive of each site) whereas $C$ shares all the 5 species present in $\mathrm{D}$ (completely nested faunas, therefore 15 species are exclusive of $\mathrm{C}$, and none of $\mathrm{D})$. Using the Sorensen index the two situations are equivalent (Similarity $\mathrm{A}-\mathrm{B}=$ Similarity $\mathrm{C}-\mathrm{D}=0.4$ ) although it is intuitively clear that turnover is greater between $A-B$ than between $C-D$, as suggests the fact 
that 32 species are involved in the first case, but only 20 in the second. Contrarily, the two cases are viewed as completely different if measured with the Simpson index (Similarity $\mathrm{A}-\mathrm{B}=0.4$; Similarity $\mathrm{C}-\mathrm{D}=1$ ). It could be argued that using the Simpson measure we are loosing the information derived of the richness differences. But this information is not lost but partitioned, since we always know richness values for each sampling site, thus richness patterns may be analyzed independently. Taking into account these considerations, when we are interested in the turnover patterns of samples involved in richness gradients, i.e. latitudinal diversity gradients, the two aforementioned situations should be discriminated in order to disentangle two independent phenomena (Harrison et al. 1992): 1) the loss of species in higher latitudes, and 2) the change in species composition between different communities. Both phenomena are related with geographic distance but the first one is only related to latitudinal distances, whereas the second is isotropic. Therefore, the integration of the two processes in the same measure yields meaningless results when comparing geographic distances necessary to reduce a fixed amount of faunal similarity, as Soininen et al. did. I am not claiming the uselessness of the Sorensen index per se, since the selection of one or another type of similarity index depends on the hypothesis to be tested. However, the questions addressed by Soininen et al. (2007) are referred, as they state in the introduction, to the second phenomenon: the change in species composition due to niche-based processes (Whittaker 1960, Nekola and White 1999), spatial limitations (Qian et al. 2005) or neutral processes as random dispersal or speciation (Hubbell 2001). For this reason, richness gradients should be controlled for, in order to test the hypothesis of the influence of variables covariating with richness (as latitude, trophic level or body size) on the distance decay of similarity.

\section{A real example}

The reanalysis of the data used by Soininen et al. (2007) is not possible because their data are the published regression coefficients between faunal similarity (computed with the Sorensen metric) and geographic distance. Since the original papers do not provide the raw data, no other turnover measures can be computed. For this reason, to illustrate the point raised here, I exemplify the different performance of similarity indices using a database of European longhorn beetles (Coleoptera: Cerambycidae) (Danilevsky 2007) and testing for the influence of the index in our perception of faunal turnover at different latitudes. Excluding islands to avoid the effect of insularity, I ordered European countries by their mean latitude, and built two different datasets of northern and southern countries of similar number of cases (19 vs 18), being the northern limits of the southern dataset France, Switzerland, Austria, Hungary, Romania and Moldova. Faunal similarity matrices were computed for the two datasets, using two different turnover measures: Sorensen (affected by richness) and Simpson (not affected) (Koleff et al. 2003). Geographic distances between countries were computed as the Euclidean distance between UTM centroids of each country. Thereafter, the relation between faunal similarity (Sorensen or Simpson metric) and geographic distance was computed applying regression on distance matrices (Legendre et al. 1994). The slope of this regression is the parameter determining the halving distance (i.e. the geographic distance needed to halve the initial similarity, in the terms of Soininen et al. 2007): the steeper the slope, the faster the distance decay of similarity, and the smaller the halving distance. To evaluate the performance of Sorensen and Simpson measures, I compared the slopes yielded by each index in the northern and southern datasets, respectively. Since the lack of independence of observations (distances or similarities) precludes the test for significance by means of traditional regression procedures, significance of the relations was computed in R (Anon. 2006) using the vegan package (Oksanen et al. 2007) by means of Mantel permutation tests. To compare the slopes yielded by the different similarity indices, the dispersion of the regression coefficients was estimated by bootstrapping, in order to detect significant differences between measures. A frequency distribution of 1000 slopes was retrieved by bootstrapping, using PopTools software (Hood 2006). The probability of obtaining the opposite result by chance was empirically computed by comparing the estimated distributions.

Using the Sorensen metric, as Soininen et al. (2007) did, the Pearson correlation coefficient between similarity and geographic distance was almost equal in the northern $(\mathrm{r}=-0.54, \mathrm{p}<0.001)$ and southern $(\mathrm{r}=$ $-0.55, \mathrm{p}=0.001)$ datasets, whereas the Simpson index produced a quite different result, being the correlation in the northern dataset $(r=-0.42, p<0.001)$ lower than in the southern dataset $(r=-0.58, p<0.001)$. Moreover, the slopes of the regressions are quite different depending on the similarity metric. In the southern dataset (Fig. 1a) the slopes (similarity $\mathrm{km}^{-1}$; mean $\pm S E)$ yielded by Sorensen $(-1.04 \mathrm{E}-04 \pm 1.19 \mathrm{E}-$ $05)$ and Simpson $(-9.59 \mathrm{E}-05 \pm 1.14 \mathrm{E}-05)$ indices are not significantly different (probability of having found the opposite result by chance, $\mathrm{p}=0.333$; Fig. 1c), whereas in the northern dataset (Fig. 1b) the slope yielded by Sorensen index $(-9.42 \mathrm{E}-05 \pm 1.14 \mathrm{E}-05)$ is almost two times the slope yielded by Simpson $(-5.70 \mathrm{E}-05 \pm 8.30 \mathrm{E}-06)$, a difference achieving high significance $(\mathrm{p}=0.008$; Fig. $1 \mathrm{~d})$. 



Southern dataset

Geographic distance $(\mathrm{Km})$

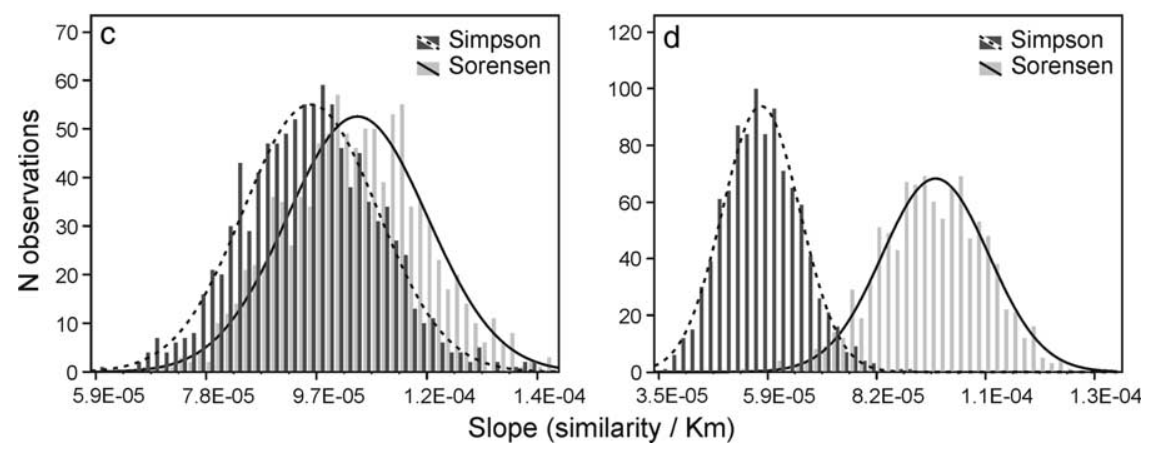

Fig. 1. Relationship between faunal similarity (Sorensen or Simpson measures) and geographic distances in the (a) southern and (b) northern datasets. The dispersion of the regression coefficients was estimated by bootstrapping in order to detect significant differences between measures. The distributions (and adjusted normal curves) of the slopes yielded by Sorensen and Simpson indices are shown for the (c) southern and (d) northern datasets.

Therefore, avoiding the confusing effect of richness gradients by means of the Simpson measure, the results disagree with those obtained by Soininen et al. (2007) and are in complete accordance with Rapoport's rule: in the northern dataset the decay of similarity with geographic distance is much slower. Thus, the result found by Soininen et al. (2007) seems to be an artefact created because the Sorensen index incorporates the differences in richness between samples. This fact is clearly illustrated by the strong negative correlation found between Sorensen similarities and species richness differences between pairs of countries, in the southern $(r=-0.570, p<0.001)$ and especially in the northern $(\mathrm{r}=-0.749, \mathrm{p}<0.001)$ dataset. In contrast, Simpson similarities presented a positive correlation with richness differences in the northern dataset $(r=0.561, p<0.001)$, and no significant relationship in the southern dataset $(\mathrm{r}=0.107, \mathrm{p}=$ $0.165)$. The positive correlation found in the northern dataset implies that in this case countries with more different species richness presented more similar composition.
In conclusion, measuring turnover with the Sorensen index, I have found similar slopes in the north and the south, with a non-negligible probability of finding a faster decay of similarity in the north $(p=0.288)$. Probably for this reason Soininen et al. (2007) found a slightly faster decay of similarity at higher latitudes. However, the Simpson measure yields a clearly faster decay of faunal similarity in the south (probability of having found the opposite result by chance, $p=0.002$ ). Therefore, the selection of an index which actually measures turnover between sites, and not the richness variation, is a crucial topic if the turnover patterns are to be understood. Richness gradients related to latitude (Whittaker et al. 2001), body size (Ulrich 2006) or trophic level (Petchey et al. 2004) are pervasive phenomena that have been widely documented. For this reason, such richness gradients should be removed from the analyzed turnover patterns if we want to disentangle both processes. Although the topic is by no means a new idea (Wilson and Shmida 1984, Harrison et al. 1992, Williams et al. 1999, Koleff et al. 2003), it seems still necessary to emphasize the importance of an 
appropriate selection of the similarity measure, since the consequences are noteworthy, and the derived patterns might even be confusing.

Acknowledgements - I wish to thank M. Danilevsky for making freely available the check-list of European Cerambycidae, and L. M. Carrascal, A. Jiménez-Valverde, R. Jovani, P. Legendre, J. M. Lobo, M. Palmer, P. Peres-Neto and three anonymous reviewers for valuable comments and suggestions on the statistical procedures or the text. Author position at MNCN is supported by Juan de la Cierva Program (Spanish Ministry of Education and Science).

\section{References}

Anon. 2006. R: a language and environment for statistical computing. $-<$ http://www.r-project.org $>$.

Danilevsky, M. L. 2007. A check-list of longicorn beetles (Coleoptera, Cerambycoidea) of Europe. $-<$ www.ceram bycidae.narod.ru $>$.

Harrison, S. et al. 1992. Beta-diversity on geographic gradients in Britain. - J. Anim. Ecol. 61: 151-158.

Hood, G. M. 2006. PopTools ver. 2.7.5. - < http://www. cse.csiro.au/poptools $>$.

Hubbell, S. P. 2001. The unified neutral theory of biodiversity and biogeography. - Princeton Univ. Press.

Koleff, P. et al. 2003. Measuring beta diversity for presenceabsence data. - J. Anim. Ecol. 72: 367-382.

Legendre, P. et al. 1994. Modeling brain evolution from behavior - a permutational regression approach. - Evolution 48: 1487-1499.

Montoya, D. et al. 2007. Contemporary richness of holarctic trees and the historical pattern of glacial retreat. - Ecography 30: 173-182.
Nekola, J. C. and White, P. S. 1999. The distance decay of similarity in biogeography and ecology. - J. Biogeogr. 26: 867-878.

Oksanen, J. et al. 2007. vegan: community ecology package. - R package version 1.8-5, < http://cran.r-project.org/ > .

Petchey, O. L. et al. 2004. Species loss and the structure and functioning of multitrophic aquatic systems. - Oikos 104: 467-478.

Qian, H. et al. 2005. Beta diversity of angiosperms in temperate floras of eastern Asia and eastern North America. - Ecol. Lett. 8: 15-22.

Reyjol, Y. et al. 2007. Patterns in species richness and endemism of European freshwater fish. - Global Ecol. Biogeogr. 16: 65-75.

Soininen, J. et al. 2007. The distance decay of similarity in ecological communities. - Ecography 30: 3-12.

Svenning, J. C. and Skov, F. 2007. Ice age legacies in the geographical distribution of tree species richness in Europe. - Global Ecol. Biogeogr. 16: 234-245.

Ulrich, W. 2006. Body weight distributions of European Hymenoptera. - Oikos 114: 518-528.

Whittaker, R. H. 1960. Vegetation of the Siskiyou Mountains, Oregon and California. - Ecol. Monogr. 30: 280338.

Whittaker, R. J. et al. 2001. Scale and species richness: towards a general, hierarchical theory of species diversity. - J. Biogeogr. 28: 453-470.

Whittaker, R. J. et al. 2007. Geographical gradients of species richness: a test of the water-energy conjecture of Hawkins et al. (2003) using European data for five taxa. - Global Ecol. Biogeogr. 16: 76-89.

Wilson, M. V. and Shmida, A. 1984. Measuring beta diversity with presence absence data. - J. Ecol. 72: 1055-1064.

Williams, P. H. et al. 1999. Interpreting biogeographical boundaries among Afrotropical birds: spatial patterns in richness gradients and species replacement. - J. Biogeogr. 26: 459-474. 